\title{
Theoretical Breakthroughs
}




\title{
OPAL Opacities
}

\author{
F. J. Rogers and C. A. Iglesias \\ Lawrence Livermore National Laboratory \\ Livermore, California 94550
}

\begin{abstract}
We have continued to improve and update the OPAL opacity code. Addition of intermediate coupling has further increased the opacity over earlier $L S$ coupling results. A 'corresponding states' method has been used to extend the tables in both $X$ and $Z$. This has allowed the calculation and distribution of extensive opacity tables for several different sets of metal abundance.
\end{abstract}

\section{Introduction}

The opacity of matter is crucial for studies of stellar evolution and pulsation. Recent improvements in opacity calculations have led to the resolution of a number of longstanding problems. For example, the mechanism for pulsation in $\beta$ Cephei stars has been identified (Cox et al. 1992; Kiriakidis, El Eid \& Glatzel 1992; Moskalik \& Dziembowski 1992) and the "bump" and "beat mass" discrepancies in Cepheid variables has been removed (Moskalik, Buchler \& Marom 1991). Many different mechanisms were introduced over the years to explain these discrepancies, but the resolution has proven ultimately to depend on improved input physics.

The success of the new OPAL opacities in the resolution of these problems has encouraged us to introduce additional improvements in the calculations and to produce updated and expanded tables. The main physics improvement has been the inclusion of spin-orbit effects in the atomic physics calculations. Furthermore, in order to facilitate the calculation of large databases we have developed a corresponding states method. We have also studied the effect of reducing the number of components in the mixture by combining the lesser abundant elements with prominent elements. A brief description of recent developments is given in the following sections.

OPAL is a completely new code having improved equation of state and atomic physics compared to that used to calculate the Los Alamos Opacity Library (Huebner et al. 1977, LAOL). It is also different in many respects from the Opacity Project (OP) (Seaton, this volume; 1987; Yu 1992). The equation of state is obtained from a many-body expansion of the grand canonical partition function (Rogers 1991) and the atomic physics is obtained from parametric potentials (Rogers, Wilson \& Iglesias 1988). The atomic structure calculations are done on-line and have accuracy similar to single-configuration Hartree-Fock with relativistic corrections. Important 
improvements in the broadening of spectral lines, inverse bremsstrahlung, and Thomson scattering have also been made. A more detailed description of OPAL is given in Rogers and Iglesias (1992a).

\section{Spin orbit interaction}

The Los Alamos opacity codes use a detailed configuration accounting method (DCA) to treat bound-state absorption (Cox \& Stewart 1965; 1970a; 1970b; Cox \& Tabor 1976, Huebner 1986). Introduction of term splitting in the $L S$ coupling scheme was responsible for a major part of the enhanced opacity obtained by OPAL compared to LAOL in the few hundred thousand degree range (Iglesias, Rogers \& Wilson 1987; 1990; Iglesias \& Rogers 1991a,b; Rogers \& Iglesias 1992a). LS coupling is valid for low- $Z$ elements where the electrostatic energy dominates. The spin-orbit interaction is very small for $Z<10$, but increases with increasing $Z$ leading to lifting of the $J$ degeneracy and to the appearance of intercombination lines. The coupling in this case is intermediate between pure $L S$ and pure $j j$. For intermediate- $Z$ elements, such as iron, the spin-orbit interaction produces small, but important effects. The different coupling approaches are illustrated in Figure 1 for the case of transitions of the type $s p$ to $p 2$. In the DCA approach, there exists a single line. The $L S$ coupling splits this line into three components corresponding to singlets and triplets. In intermediate coupling, the spin-orbit effect further splits the spectrum into 8 lines having $\Delta S=0$ and 6 intercombination lines; 3 lines having $\Delta S=+1$, and 3 having $\Delta S=-1$. For more complicated configurations, the increase in the number of distinct lines can be much larger.

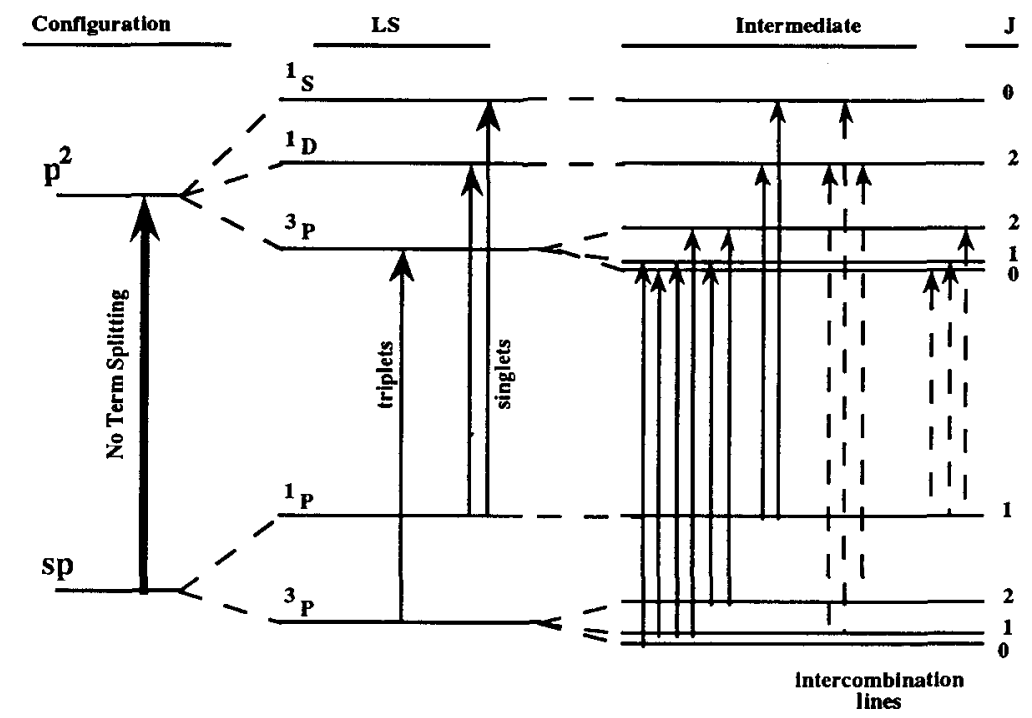

Figure 1. Schematic drawing showing various angular coupling schemes. 
The splitting into additional lines distributes the oscillator strength more uniformly over frequencies and is similar to increasing the line width. The introduction of intermediate coupling can, thus, further increase the opacity when the spin-orbit "broadening" exceeds the line broadening. Since the spin-orbit effect does not depend on density, while line broadening does, its effects become more important at low density. This is illustrated in Figures $2(\mathrm{a}-\mathrm{c})$, which compares the OPAL intermediatecoupling result to the $L S$ coupling result for several values of $R$ vs. $T$ ( $R=\rho / T_{6}^{3}$ and $\left.T_{6}=T / 10^{6} \mathrm{~K}\right)$. The intermediate-coupling scheme gives an enhancement of $50 \%$ at $\log R=-5$ and $T_{6}=0.25$, whereas the enhancement is insignificant at $\log R=-2$. A more complete description of this work is given in Iglesias, Rogers \& Wilson (1992).
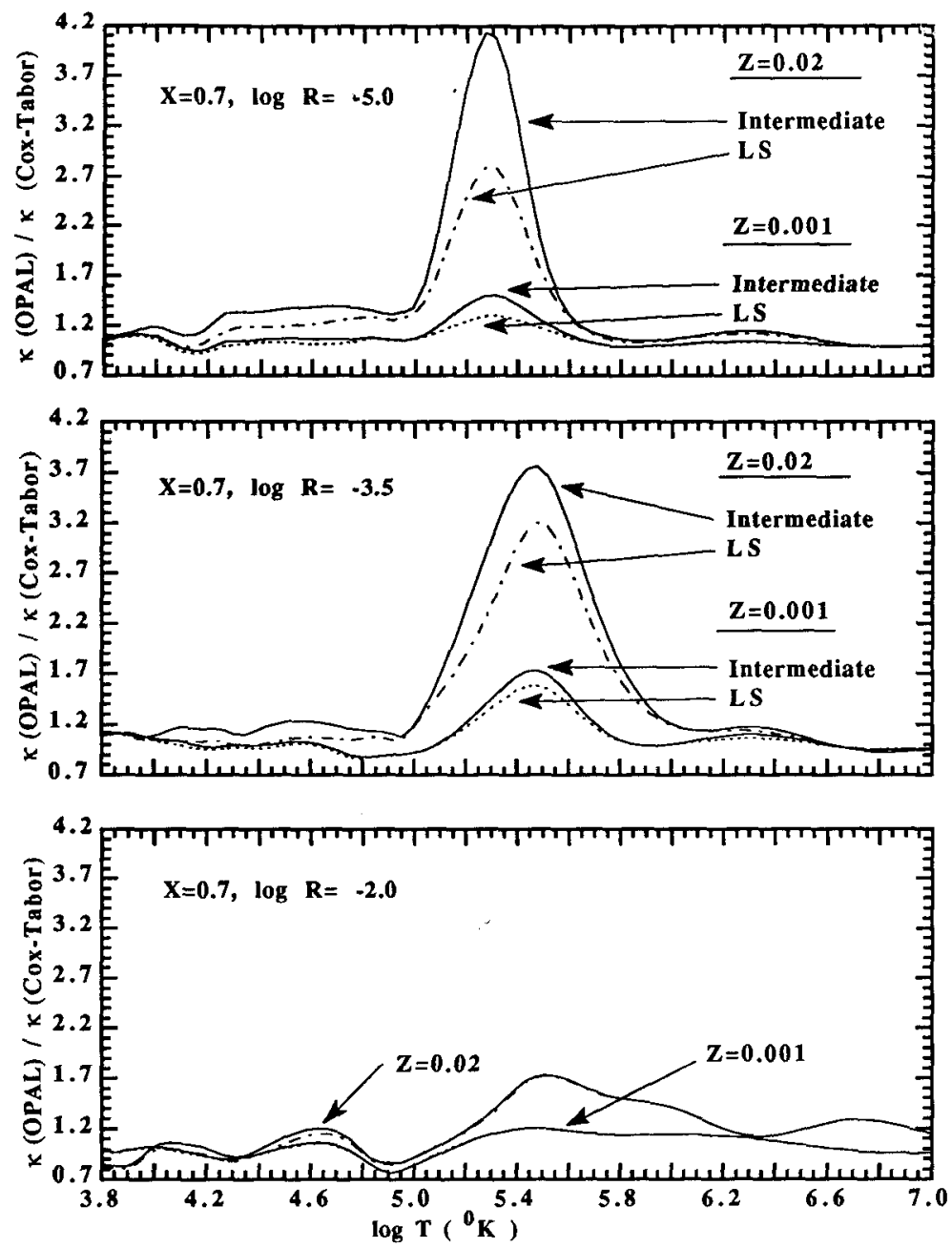

Figure 2. Intermediate coupling effects on the Rosseland mean opacity. 


\section{Corresponding States}

The Los Alamos Opacity Library is a compilation of single-element calculations at specific values of $T$ and electron chemical potential, $\mu_{e}$. The photoabsorption coefficient for a mixture is obtained by adding these individual element cross-sections at fixed $T$ and $\mu_{e}$ weighted by their abundance. The Rosseland mean opacity is then obtained by integrating the total over photon energy and the density of the resulting mixture is obtained from the ideal gas law of additive volumes.

In our work we calculate the equation of state for the full mixture, including the Coulomb coupling between the various constituents. Consequently, we cannot use the LAOL approach for mixing. However, it is possible to show that the intra-ionic ratios for ions of type $i$ in the various states of ionisation can be the same for mixtures having different elemental mass fractions, $\chi_{i}$, and densities. The conditions for this to occur is that the temperature and electron number density be the same. It follows that a solution for the equation of state for a mixture characterised by $T, n_{e}, \rho$, and $\chi_{i}$ can be used to find the equation of state for a different mixture characterised by $T, n_{e}, \rho^{\prime}$, and $\chi_{i}^{\prime}$. The electron density for the initial and final mixtures are given by

$$
n_{e}=\rho \sum Q_{i}^{*} \chi_{i} / A_{i} \text { and } n_{e}=\rho^{\prime} \sum Q_{i}^{*} \chi_{i}^{\prime} / A_{i}
$$

where $Q_{i}^{*}$ is the average state of ionization for element $i$ and $A_{i}$ is the atomic weight. The densities of the two mixtures are thus related according to

$$
\rho^{\prime}=\rho\left\{\sum Q_{i}^{*} \chi_{i} / A_{i}\right\}\left\{\sum Q_{i}^{*} \chi_{i}^{\prime} / A_{i}\right\}^{-1}
$$

Since the relative intra-ionic occupation numbers are the same in the two mixtures, it is possible to rapidly calculate the opacity of the second mixture from the data already calculated and stored for the first mixture. We have used this approach to expand the tables in $X$ and $Z$ (Rogers \& Iglesias 1992b). The current tables cover 10 values of $X$ in the range 0 to $1-Z$ and 13 values of $Z$ in the range 0 to 0.1 .

Figure 3 shows $\log \kappa_{R}$ vs. $\log T_{6}$ for the extremes of $X$ when $Z=0.02$. A number of differences related to the $\mathrm{H}$ abundance in the mixture are apparent. There is a rapid rise in opacity at low temperatures which is caused by the ionisation of hydrogen when $X=1-Z$ and to the ionisation of helium when $X=0$. In the latter, the higher ionisation potential of He shifts the onset of this rapid increase in $\kappa_{R}$ towards higher temperatures. The opacity bump occurring near $\log T_{6}=-0.7$ is more pronounced for $X=0$ and is due, in part, to the photoionisation of the $K$-shell electron in singly-ionized He. In addition, there are relatively more metals by number in the $X=0$ mixture, further enhancing the bump near $\log T_{6}=5.2$. Finally, the lower electron density of the $X=0$ composition leads to lower photon scattering and free-free absorption contributions; consequently, its opacity is lower at high temperatures. 


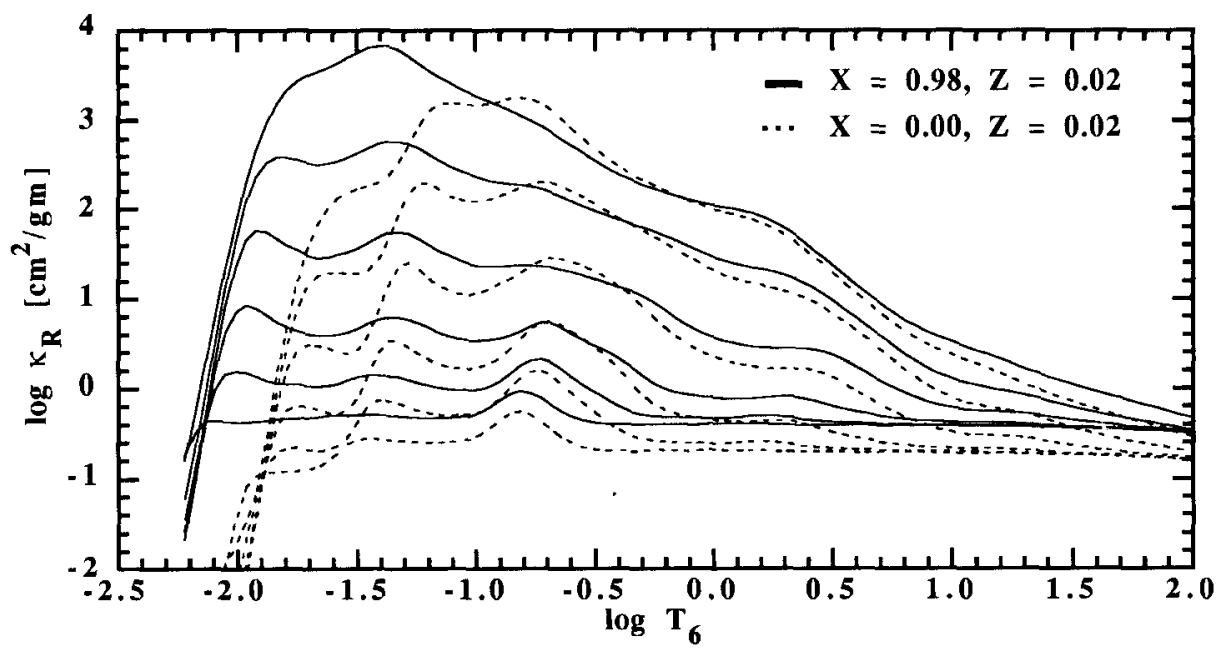

Figure 3. Comparison of $\kappa_{R}$ for $X=0$ and $1-Z$. The curves are for constant $R$ values: (from top to bottom) $\log R=-1,-2,-3,-4,-5$, and -7 .

Due to the versatility of the 'corresponding states' method we can use the stored occupation number and photoabsorption data to rapidly calculate opacity tables covering the same range of $X$ and $Z$ for arbitrary heavy-element abundance. We are currently producing tables for advanced-type stars that have enriched $\mathrm{He}, \mathrm{C}$, and $\mathrm{O}$ abundance.

\section{Abundance Effects}

Metal abundance affects opacities in both fundamental and practical ways. The former arise from observational and calculational uncertainties in elemental number fractions. The latter results from the need to conserve computer time. Since some elements have very low abundance compared to their neighbors, it is common practice to lump the abundance of these elements with the more abundant neighbor. The opacity tables given in Rogers \& Iglesias (1992a) were for $\mathrm{H}, \mathrm{He}$, and a twelve-element composition for $Z$. In particular, we added $\mathrm{Cr}$ and $\mathrm{Ni}$ together with $\mathrm{Fe}$. Figure 4 shows the ratio of the OPAL opacities vs. temperature at $\log R=-5$ for a 16element mixture that treats $\mathrm{Cr}, \mathrm{Fe}$, and $\mathrm{Ni}$ as distinct components in contrast to the 14-element mixture in which $\mathrm{Cr}$ and $\mathrm{Ni}$ have been combined with $\mathrm{Fe}$. A substantial enhancement of about $28 \%$ occurs around $T_{6}=0.25$. Similar results have also been obtained by the Opacity Project (Seaton, this volume). Even so, this enhancement is small compared to the factor of 4 obtained over LOAL with the introduction of $L S$ coupling. 


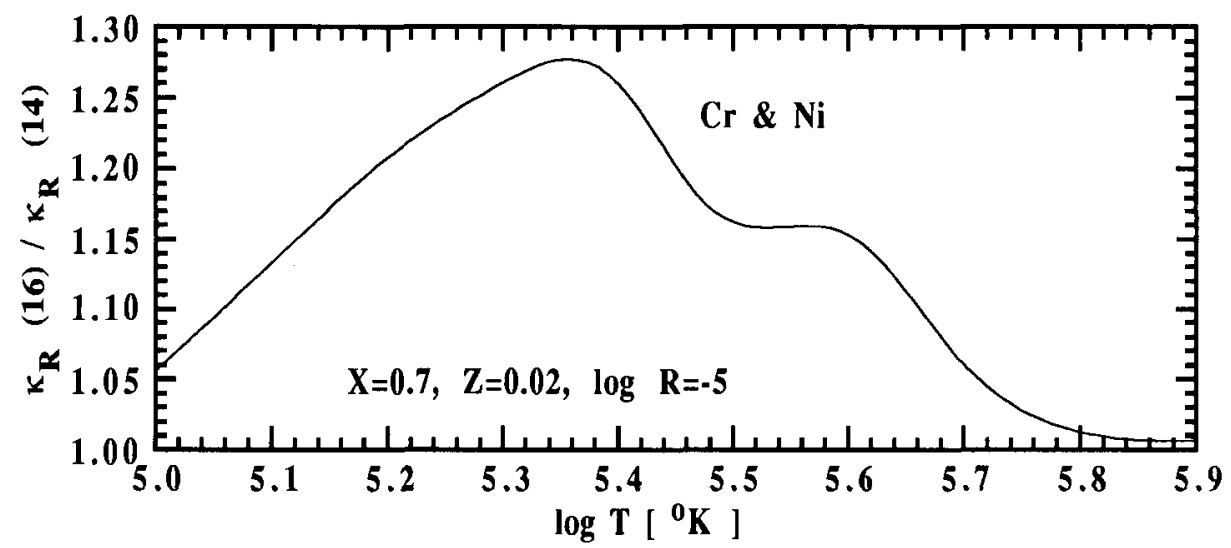

Figure 4. Effect of including $\mathrm{Cr}$ and $\mathrm{Ni}$ explicitly in mixture.

Figure 5 gives the frequency-dependent absorption cross-section for $\mathrm{Cr}, \mathrm{Fe}$, and $\mathrm{Ni}$ at $T_{6}=0.2$ and $\log R=-5$. Due to the $Z^{2}$ scaling of the energies, valleys in the $\mathrm{Fe}$ absorption are filled in by strong features in the $\mathrm{Cr}$ and $\mathrm{Ni}$ spectrum. In such cases, it is not a good approximation to treat the $\mathrm{Cr}$ and $\mathrm{Ni}$ as though their spectrum is the same as that of Fe. This was also noted earlier by Rogers \& Iglesias (1992a), where only the enhancement due to $\mathrm{Ni}$ was considered. Revised tables including $\mathrm{Cr}$ and $\mathrm{Ni}$ are being calculated.

\section{Experimental verification}

Measuring the opacity at stellar interior conditions is very difficult and the capability has only recently been developed (Perry et al. 1991). The procedure involves use of two laser beams, one to heat a thin metal sample up to several hundred thousand degrees and the other to act as a backlighter source. The measured transmission of the backlighter source, $e^{-\rho L \kappa(\nu)}$, where $\rho$ is the sample density and $L$ the sample thickness, yields the frequency-dependent opacity, $\kappa(\nu)$.

The recent OPAL calculations predict large enhancements to astrophysical opacities due to transitions in the $M$-shell of iron; particularly the $\Delta n=0$ lines around $70 \mathrm{eV}$. So far two experiments have been completed that test the validity of the OPAL code for treating absorption in iron. The first (Da Silva et al. 1992) studied pure Fe at $\log T \simeq 5.4$ and $\rho \simeq 0.008 \mathrm{~g} / \mathrm{cm}^{3}$ and was designed to observe the prominent $\Delta n=0$ features. Since the calculations predict that these features persist over a wide range of temperature and density, the diagnostics in the experiment were not designed to make accurate measurements of plasma conditions. As a result, the temperature uncertainty is $20 \%$ and the density is estimated to within a factor of two. 

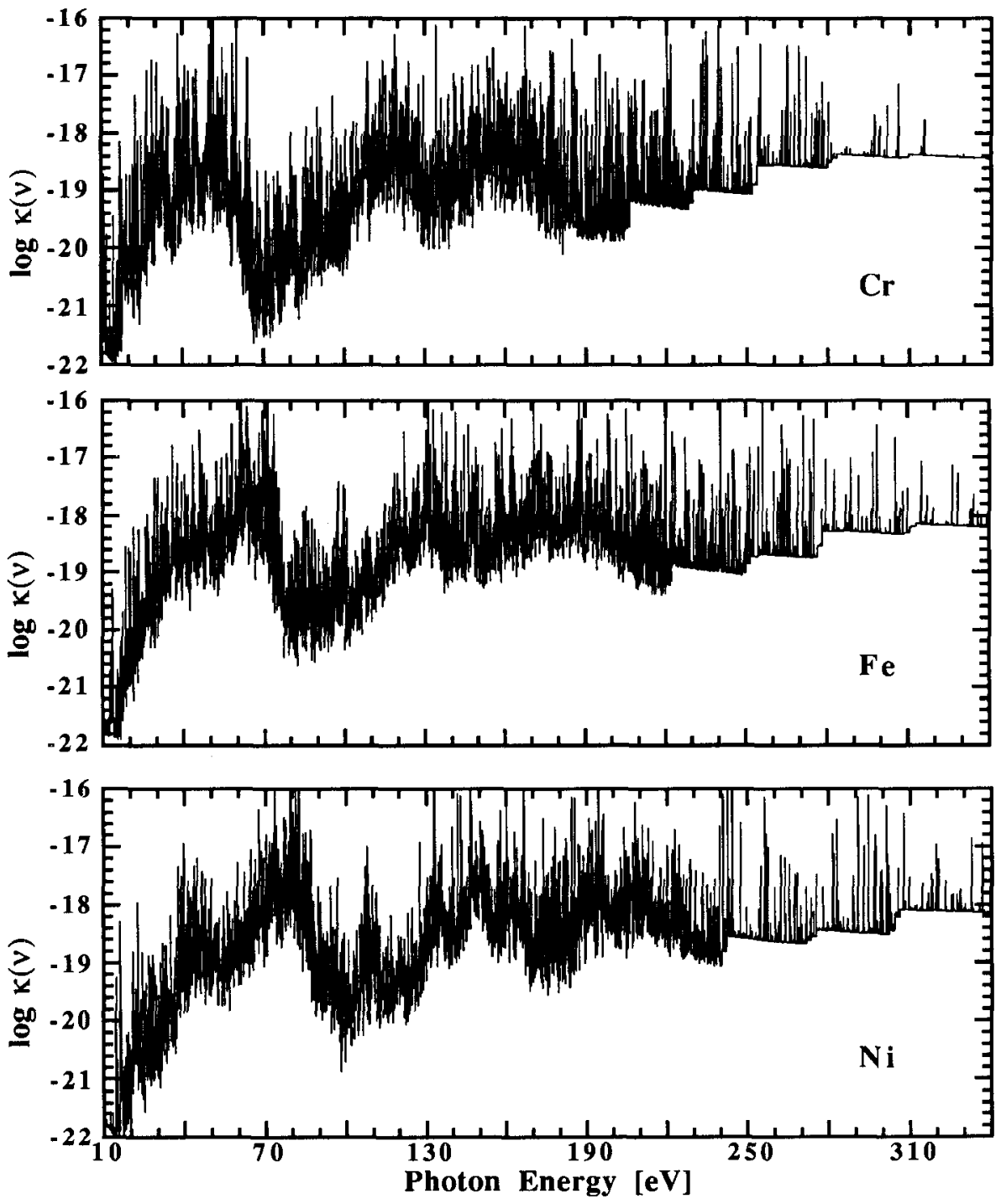

Figure 5. Photoabsorption coefficients $\left(\mathrm{cm}^{2} /\right.$ nuclei) for $\mathrm{Cr}, \mathrm{Fe}$, and $\mathrm{Ni}$ for a plasma at $\log T=5.3$ and $\log R=-5$. Note similarity, but also small shift towards higher photon energy with increasing nuclear charge.

The results of the Da Silva et al. experiment are compared with two versions of OPAL in Figure 6. It is apparent that the DCA calculation, which approximates the LAOL calculations, has no absorption features in the crucial $70 \mathrm{eV}$ photon energy range; whereas both the OPAL calculation with full intermediate coupling and the experiment show strong absorption features in this range. The experiment thus offers confirmation that the OPAL prediction is qualitatively correct. 


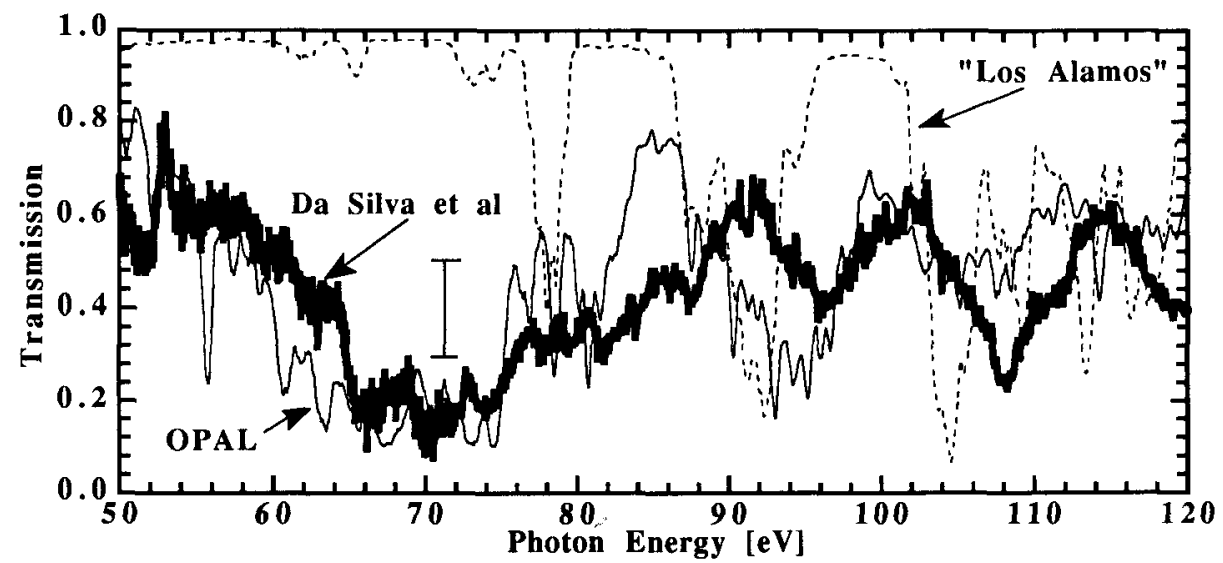

Figure 6. The Fe transmission experiment (heavy solid line) is compared to OPAL with intermediate coupling (thin solid line) and OPAL in DCA with hydrogenic oscillator strengths (dashed line) simulating the LAOL calculations. Experimental error bars for the transmission are indicated in the figure.

The second experiment (Springer et al. 1992) introduces a small impurity of $\mathrm{NaF}$ in the $\mathrm{Fe}$ sample in order to determine the plasma temperature using an established method (Perry et al. 1991). They also developed a technique to determine the density accurately. The purpose of this experiment was to measure the frequencydependent absorption over the energy range that contributes most to the Rosseland mean in a well-characterised experiment; the first such experiment in a hot plasma. The conditions were determined to be $k T=59 \pm 3 \mathrm{eV}$ and $\rho=0.0135 \pm 0.0014$ $\mathrm{g} / \mathrm{cm}^{3}$. Integration over the experimentally obtained $\kappa(\nu)$ gave $\kappa_{R}=4400 \pm 600$ $\mathrm{cm}^{2} / \mathrm{g}$. The OPAL result for nominal conditions is $4100 \mathrm{~cm}^{2} / \mathrm{g}$. This experiment, thus, also supports the OPAL results.

\section{Conclusion}

We have continued to improve and update the OPAL opacity calculations. Substantial increases in opacity over our earlier work were obtained with the introduction of intermediate coupling; particularly at low density where the spin orbit splitting can be greater than the spectral line broadening. Significant increases in opacity were also obtained by explicitly including the lowly abundant $\mathrm{C}_{r}$ and $\mathrm{Ni}$ in the metal mixture; clearly showing that even elements of very low abundance can make important contributions when their spectrum fills in valleys where no other element has absorption features. This again demonstrates that accurate opacities also require an accurate determination of the stellar element composition.

The use of OPAL opacities has led to the resolution of a number of long-standing 
discrepancies between theory and observation in pulsating stars. The OPAL opacities have also improved the agreement with observation on a number of other problems (Cox 1991; Kovacs, Buchler \& Marom 1991; Stothers 1992; Stothers \& Chin 1992; El Eid \& Hartman 1992). Recent laser experiments on thin metal foils have also corroborated the large $\Delta n=0$ contribution from $M$-shell iron and verified the Rosseland mean at one temperature-density point. This is strong evidence that the OPAL opacities represent a significant improvement over LAOL. The close agreement of OP and OPAL (Seaton, this volume) in the range where OP is valid $\left(\rho<0.01 \mathrm{~g} / \mathrm{cm}^{3}\right)$ is additional strong evidence supporting both efforts.

We are indebted to B.G. Wilson for the angular momentum coupling code in the atomic data generation and R.W. Lee for his linear Stark broadening subroutines. Additional thanks are due to R.W. Lee for providing us with the experimental Fe transmission data. Work performed under the auspices of the U.S. department of Energy by the Lawrence Livermore National Laboratory under contract W-7405-Eng48.

\section{References:}

Cox, A. N. 1991, ApJ, 381, L71

Cox, A. N., Morgan, S. M., Rogers, F. J., \& Iglesias, C. A. 1992, ApJ, 392, 272

Cox, A. N., \& Stewart, J. N., 1965, ApJS, 11, 22

-. 1970a, ApJS, 19, 243

-. 1970b, ApJS, 19,261

Cox, A. N., \& Tabor, J. E. 1976, ApJS, 31, 271

Da Silva, L. B., MacGowan, B. J., Kania, D. R., Hammel, B. A., Back, C. A., Hsieh, E., Doyas, R., Iglesias, C. A., Rogers, F. J., \& Lee, R. W. 1992, Phys. Rev. Lett., 69, 438

El Eid, M. F. \& Hartman, D. H., 1992, ApJL (submitted)

Huebner, W. F. 1986, Physics of the Sun, Vol. 1, ed. P. A. Sturrock, E. Holzer, D. M. Mihalas, \& R. K. Ulrich (Dordrecht: Reidel), p. 33

Huebner, W. F., Merts, A. L., Magee, N. H., \& Argo, M. F. 1977, Los Alamos Scientific Report LA-6760-M

Iglesias, C. A., \& Rogers, F. J. 1991a, ApJ, 371, 173

$-.1991 \mathrm{~b}, \mathrm{ApJ}, 371,408$

Iglesias, C. A., Rogers, F. J., \& Wilson, B. G. 1987, ApJ, 322, L45

-. 1990, ApJ, 360, 221

-. 1992, ApJ (October)

Kiriakidis, M., El Eid, M. F., \& Glatzel, W. 1992, MNRAS (Letters), 255, 1

Kovacs, G., Buchler, J. R., \& Marom, A. 1991, A\&A, 25, 685

Moskalik, P., \& Dziembowski, W. A. 1992, A\&A, 256, L5 
Perry, T. S., Davidson, S. J., Serduke, F. J. D., Bach, D. R., Smith, C. C., Foster, J. M., Doyas, R. J., Ward, R. A., Iglesias, C. A., Rogers, F. J., Abdallah, Jr., J., Stewart, R. E., Kilkeny, J. D., Lee, R. W. 1991, Phys. Rev. Lett., 67, 3784

Rogers, F. J. 1991, in High Pressure Equations Of State: Theory and Applications, ed. S. Eliezer \& R. A. Ricci (North Holland, New York, 1991)

Rogers, F. J., \& Iglesias, C. A. 1992a, ApJS, 79, 507

一. 1992b, ApJ (December)

Seaton, M. J. 1987, J. Phys. B, 20, 6263

Springer, P. T., Fields, D. F., Wilson, B. G., Nash, J. K., Goldstein, W. H., Iglesias, C. A., Rogers, F. J., Swenson, J. K., Chen, M. H., Bar-Shalom, A., \& Stewart, R. E. 1992, Phys. Rev. Lett. (submitted)

Stothers, R. B., \& Chin, C.-W., 1992, ApJ, 390, 136

Stothers, R. B. 1992, ApJ, 392, 706

Swenson, F. J., Stringfellow, G., \& Faulkner, J. 1990, ApJ, 348, L33

Yu, Y. 1992, Rev. Mex. Astron. Astrof., 23, 171 